\title{
Adolescência conectada: Mapeando o uso da internet em jovens internautas ${ }^{1}$
}

\author{
Adolescence connected: Mapping out the use of internet in young users of the Internet
}

\author{
Rosane Cristina Pereira Spizzirri ${ }^{[a]}$, Adriana Wagner ${ }^{[b]}$, Clarisse Pereira Mosmannn ${ }^{[\mathrm{c}]}$, Ananda Borgert Armani ${ }^{[\mathrm{d}]}$
}

${ }^{[a]}$ Mestre em Psicologia Social Pontifícia Universidade Católica do Rio Grande do Sul (PUCRS), Porto Alegre, RS - Brasil, e-mail: rosane.spiz@terra.com.br

${ }^{[b]}$ Doutora em Psicologia Universidade Federal do Rio Grande do Sul (UFRGS), Porto Alegre, RS - Brasil, e-mail: adrianawagner.ufrgs@hotmail. com

${ }^{[c]}$ Doutora em Psicologia Universidade Federal do Rio Grande do Sul (UFGRS), Porto Alegre, Brasil. Porto Alegre, RS - Brasil, e-mail: clarisse@redemeta.com.br

${ }^{[d]}$ Estudante de Psicologia Pontifícia Universidade Católica do Rio Grande do Sul (PUCRS), Porto Alegre, RS - Brasil, e-mail: naniarmani@hotmail.com

Recebido: $25 / 08 / 2010$

Received: 08/25/2010

Aprovado: 04/05/2011 Approved: 05/04/2011

\section{Resumo}

A crescente propagação de estudos que enfocam os comportamentos e o envolvimento com as novas tecnologias dos jovens que representam a denominada geração online vem suscitando questionamentos e discussões entre pesquisadores e educadores. As inúmeras indagações acerca das condutas e regras que constituem o mundo virtual por parte dos adolescentes, demonstram que nos encontramos na presença de um fenômeno ainda pouco explorado que tem gerado vários desafios para aqueles envolvidos na educação dessa geração. Diante desse panorama, este estudo buscou mapear as diferentes formas de uso da internet pelos adolescentes considerando as variáveis: sexo, idade, tipos de escola, finalidade de uso, frequência de utilização e a relação com a família. Participaram desta pesquisa 534 adolescentes de ambos os sexos, de escolas públicas e privadas com idades entre 12 e 17 anos. Utilizou-se o Questionário para Uso de Eletrônicos. Os resultados demonstraram que a grande maioria dos jovens possui computador, a maior parte da amostra utiliza a internet de duas a três horas diárias, em suas casas, com pouco ou nenhum monitoramento dos progenitores e/ou cuidadores. Conclui-se que o perfil de uso dos adolescentes caracteriza um universo particular ao qual a família tem pouco acesso tanto em relação às formas de utilização quanto ao conteúdo acessado. Neste contexto, aponta-se a necessidade de um maior envolvimento e esclarecimento de pais, educadores e profissionais da saúde a respeito da relação que os jovens vêm estabelecendo com essa tecnologia.

Palavras-chave: Internet. Adolescentes. Família.

\section{Abstract}

The increasing spread of studies that focus on behaviors and involvement of youth, representatives the so-called generation online, with new technologies has raised questions and discussions among researchers and educators. The uncountable questions about the conduct and rules of a virtual world constituted by adolescents show that we are faced with a not yet explored

10 presente trabalho deriva da dissertação de Mestrado intitulada "O uso da internet na adolescência: Aspectos relativos às relações familiares na pós-modernidade". 
phenomenon that has generated a number of challenges for those involved in education for this generation. Faced with this background this study tried to map out the different forms of Internet use by adolescents considering gender, age, school types, usage, frequency of use and relationship with the family. Participated in this study 534 adolescents of both sexes, from public and private schools, aged between 12 and 17 years old. We used the Questionnaire for Computer Use. The results showed that the vast majority of young people have computers, most of the sampled individuals use the Internet 2-3 hours daily in their homes with little or no monitoring of the parents and/or caregivers. We have come to the conclusion that adolescent usage profile characterizes a particular universe to which the family has little access regading both the ways it is used and the contents accessed. In this context, it points out the need for greater involvement and clarification of parents, educators and healthcare professionals about the relationship young people have established with this technology.

Keywords: Internet. Adolescents. Family.

\section{Introdução}

Afinal, o que tanto eles fazem na frente do computador? Difícil é encontrar quem nunca tenha questionado isto. A intensa propagação de estudos acerca de uma nova geração online e o comportamento do adolescente na atualidade tem gerado inquietantes discussões entre aqueles que são responsáveis pela orientação e educação de nossos jovens. Já está comprovado que a frequência de utilização, as formas e finalidades de uso geram a necessidade de controle no uso de tal tecnologia, graças, por exemplo, aos aspectos de segurança que envolve as relações virtuais. Diante disso, cada vez mais são bem-vindas pesquisas que revelem as peculiaridades e características desse fenômeno.

Algumas iniciativas no contexto brasileiro revelam a intensidade acelerada com que tal tecnologia tem se disseminado entre a juventude. 0 Ibope/NetRatings (2007), realizando um levantamento quanto a frequência de utilização mundial, apontou o Brasil como o país recordista em número de usuários residenciais, ultrapassando 19,3 milhões de pessoas. Segundo Ibope/NetRatings (2008), nosso país se revelou recordista também no que se refere ao número médio de horas mensais de utilização domiciliar, atingindo $23 \mathrm{~h} 28$.

Os países com maior semelhança em tempo médio de conexão residencial, comparado aos internautas brasileiros foram o Japão (21h34), a França (20h23), os Estados Unidos (19h46) e a Austrália (18h) (Ibope/NetRatings, 2008). Assim, torna-se importante a realização de pesquisas que abarquem a utilização crescente da internet entre o público jovem, que é o mais representativo, sobretudo estudos que abordem os aspectos relacionados com a família e a percepção de apoio social e bem-estar dos adolescentes. O uso da internet na adolescência assume, portanto, significados e efeitos que merecem atenção no que diz respeito ao desenvolvimento da nova geração.

A relevância desse fenômeno também foi apontada pela ONG SaferNet Brasil (2008), responsável pela Central Nacional de Denúncias de Crimes Cibernéticos e que opera em parceria com o Ministério Público Federal de São Paulo (MPF-SP). A ONG divulgou uma pesquisa que contou com a participação de quase 1.400 crianças, jovens, pais e mães de todo o país. Um dos dados mais preocupantes revelados é de que $87 \%$ dos jovens afirmaram não possuírem restrições quanto ao uso da internet. Destes, $64 \%$ usam a internet no quarto, com total privacidade, contrariando as orientações preventivas dos especialistas que reiteram a importância de que o computador seja mantido em áreas de circulação da casa, a fim de que o uso feito pelo jovem seja possível ser monitorado pelos adultos.

\section{A utilização da internet na adolescência}

Na tentativa de caracterizar o fenômeno das relações diante da virtualidade, o sociólogo canadense Tapscott (1998) denominou esta geração como "NetGeneration", "Geração Y", "Geração digital”, entre outras denominações. Esses jovens são caracterizados 
por estarem sempre conectados a alguma mídia, muitas vezes, a mais de uma simultaneamente. Entretanto, existem muitas diferenças quanto à forma de utilização, comportamento e tipos de uso entre eles que variam de acordo com fatores sócio-econômicos, culturais, emocionais, entre outros.

Existem tentativas de conhecer as características e modos de utilização da internet, principalmente entre os jovens. Muitas pesquisas nacionais têm sido desenvolvidas nesta área (Almeida \& Eugênio, 2006; Nicolaci da Costa, 1998, 2002, 2006; SaferNet Brasil, 2008). A pesquisa Dossiê Universo Jovem-3 (MTV, 2005), que identificou um perfil dos jovens e o uso que fazem da comunicação virtual, revelou que os entrevistados afirmaram que a comunicação virtual ganhou novas linguagens e canais específicos, tornando possível selecionar o site ideal de acordo com o que e para quem se quer dizer, no tempo escolhido.

Em vitude destes aspectos, os jovens atribuem à internet uma melhora na forma de se relacionar com os amigos. Assim, a internet favorece mais liberdade de comunicação proporcionando formas alternativas de se expressar. Dos participantes, 39\% passaram a falar mais com os amigos e $48 \%$ afirmam mentir quando estão se comunicando virtualmente. Fica claro que, nesta geração de adolescentes, as fronteiras entre o mundo virtual e o mundo real são difusas. Os jovens entrevistados afirmaram que os diálogos por mensagens são tão intensos quanto um encontro físico e, muitas vezes, até mais íntimos.

Diante disso, percebe-se que a web tem sido utilizada quase como um laboratório social, capaz de proporcionar a possibilidade de testar os limites dos relacionamentos, por exemplo. Existem ainda aqueles jovens que se utilizam da internet como uma forma de superar as suas dificuldades sociais, conflitos emocionais, timidez, entre outros fatores presentes em qualquer relação.

Nesta perspectiva, pesquisas interessadas em identificar as possíveis causas da utilização excessiva da internet, encontraram como justificativas: o anonimato, a acessibilidade, a segurança e o uso fácil desta ferramenta (Young, 1998). A partir da análise dos diálogos mantidos pelos usuários, foram identificados aspectos reforçadores do uso abusivo, tais como fatores relacionados ao suporte social, à realização sexual e à possibilidade de criação de um personagem fictício. Entretanto, também foi observado que os internautas que não fazem uso excessivo justificam com os mesmos argumentos o porquê utilizam essa tecnologia que os adictos. Nesse caso, observa-se que outras variáveis estão implicadas na explicação deste fenômeno.

A partir de tal panorama, este estudo teve como objetivo mapear os modos de utilização da internet pelos adolescentes, buscando conhecer as finalidades e as formas de uso, assim como a frequência e locais onde a utilizam. Tais variáveis foram analisadas enfocando o contexto familiar dos participantes.

\section{Método}

\section{Amostra}

A amostra foi constituída de 534 adolescentes com idades entre 12 e 17 anos, sendo 242 do sexo masculino e 292 do sexo feminino. A média de idade dos sujeitos foi de 14,23 anos ( $d p=1,614)$, sendo que a maior concentração ocorreu entre os 13 e 14 anos, conforme a Tabela 1:

\section{Tabela 1 - Distribuição da amostra conforme a idade}

\begin{tabular}{ccc}
\hline Idade & Frequência & \% \\
\hline 12 & 90 & 17,6 \\
$\mathbf{1 3}$ & $\mathbf{1 0 5}$ & $\mathbf{2 0 , 5}$ \\
$\mathbf{1 4}$ & $\mathbf{1 0 6}$ & $\mathbf{2 0 , 7}$ \\
15 & 70 & 13,7 \\
16 & 88 & 17,2 \\
17 & 52 & 10,2 \\
\hline
\end{tabular}

Fonte: Dados da pesquisa.

Quanto ao grau de escolaridade, eram alunos da sexta série do ensino fundamental ao terceiro ano do ensino médio de escolas estaduais (27\%), federais $(31,6 \%)$ e privadas (41\%) da cidade de Porto Alegre.

\section{Instrumento e procedimentos}

O instrumento dividiu-se em duas partes:

- Parte I: avaliou os dados sócio-bio-demográficos, nível sócio-econômico (NSE) dos pais (Hollingshead, 1975), além de informações relativas à configuração da família do adolescente;

- Parte II: (questionário para uso de eletrônicos) explorou as diferentes atividades e opiniões 
dos adolescentes sobre o uso da internet. Está composto de perguntas a respeito de locais, disponibilidade, finalidades e horas de utilização da internet. Também investiga como este uso se reflete nas relações interpessoais dos adolescentes com seus iguais, irmãos e progenitores. Instrumento traduzido e adaptado de Malo (2008).

Os sujeitos foram contatados por meio de escolas da rede de ensino público e privado da cidade de Porto Alegre. Os questionários foram aplicados no espaço escolar de forma coletiva, sendo que os adolescentes participantes foram previamente autorizados por seus pais, por meio da assinatura do Termo de Consentimento Livre e Esclarecido.

\section{Apresentaç̃̃o e discussão dos resultados}

Primeiramente, fez-se uma análise descritiva dos dados obtidos a fim de observar o comportamento das variáveis em estudo (média, mediana, moda, desvio-padrão, frequências e percentuais) e mapear o uso que os sujeitos faziam da internet. A comparação da relação entre as variáveis sexo e idade foi feita a partir do teste $T$ de Student. Utilizou-se o Chi-quadrado com a finalidade de verificar as possíveis diferenças entre as variáveis: tipo de uso da internet, sexo e idade dos sujeitos.

Por fim, utilizou-se o teste de Mann-Whithney para analisar as diferentes utilidades da internet na visão dos adolescentes, o que pensam acerca de seu uso, com quem costumam compartilhar conhecimentos a respeito da internet, o sexo do sujeito e a finalidade de seu uso. Foi realizado também o teste de Kruskall-Wallis, para observar o comportamento destas variáveis nas diferentes faixas etárias da amostra. 0 grau de significância adotado foi de 5\%.

\section{Mapeando o contexto familiar dos entrevistados}

Ao perguntar "quem mora contigo na tua casa?" foi possível mapear diferentes configurações familiares entre os participantes investigados. Mais da metade dos participantes coabita em famílias nucleares ( $\mathrm{n}=282 ; 53,4 \%)$. Alguns tendo outros irmãos ( $\mathrm{n}=190 ; 36 \%$ ) e outros sendo o único filho na casa ( $\mathrm{n}=92 ; 17,4 \%)$. Um segundo grupo co-habita em famílias monoparentais ( $\mathrm{n}=155 ; 29,4 \%)$, e outro grupo em famílias estendidas $(n=45 ; 8,5 \%)$. E a menor porcentagem dos jovens coabita em famílias reconstituídas ( $\mathrm{n}=40 ; 7,7 \%)$.

A ocupação das mães e dos pais dos participantes foi avaliada segundo a classificação de Hollingshead (1975), adaptada por Tudge e Frizzo (2002), por meio da Escala de Nível Sócio Econômico - NSE, na qual classifica-se o Nível Ocupacional - NO e o Nível de Escolaridade - NE dos pais dos participantes do estudo. O Nível Ocupacional está classificado em ocupações que demandam nível de escolaridade fundamental, (ex: do lar, desempregado, agricultor, faxineira), nível de escolaridade médio (ex: operadores de máquinas, balconistas, auxiliares de escritórios) e nível de escolaridade superior (ex: gerentes, administradores, profissionais liberais).

Quanto ao NO das mães, observa-se que existe uma parcela significativa $(42,7 \%)$ exercendo atividades profissionais que exigem curso superior, enquanto em segundo lugar $(27,2 \%)$ estão aquelas que exercem atividades com a exigência apenas de nível educacional de ensino fundamental.

Já quanto ao NO dos pais, percebe-se que 44,7\% exercem trabalhos que exigem nível educacional superior, sendo que $33,1 \%$ realizam atividade laboral de nível médio. Encontrou-se um baixo índice de pais desempregados ou do lar.

Quanto ao NE - Nível de Escolaridade, o levantamento descritivo revelou que, em relação à Pós-Graduação Completa, as mães $(24,1 \%)$ possuem um nível de escolaridade mais elevado do que os pais $(21,2 \%)$ dos adolescentes participantes. Já quanto ao ensino superior completo, os pais $(28,4 \%)$ apresentam uma leve vantagem com relação às mães $(27,6 \%)$ e o mesmo ocorre com o ensino médio completo, em que $24,9 \%$ dos pais o concluíram diante de $23,3 \%$ das mães.

Identifica-se que as configurações familiares dos entrevistados são diversificadas refletindo a realidade atual das famílias onde se encontra diferentes arranjos. Entretanto, um pouco mais da metade da amostra vive com os dois progenitores $(53,4 \%)$, em um arranjo clássico, enquanto um porcentual menor 29,4\% pertence à famílias monoparentais. Essas figuras parentais possuem escolaridade de média e superior com categoria ocupacional compatível a sua formação. 


\section{0 uso da internet: características e finalidades}

\section{Análise descritiva}

Em relação ao uso da internet, 89,6\% dos adolescentes participantes desta pesquisa têm computador com acesso à internet em casa, e 10,4\% não tem. Dentre aqueles que utilizam tal tecnologia, 50,2\% possui esse recurso há mais de quatro anos, 10,5\% de 3 a 4 anos, $12,7 \%$ de 2 a 3 anos, $10,5 \%$ de 1 a 2 anos, $6,2 \%$ menos de 1 ano, sendo que $10 \%$ não informaram. Questionados quanto aos sites de relacionamento de maior utilização, 40,6\% se comunica por meio do Orkut e do MSN (Messenger), sendo que estes foram classificados na seguinte ordem de preferência 87,64\% MSN, 70,22\% Orkut, 25,28\% e-mail, $3,37 \%$ chats.

Em relação ao tempo de utilização, 38,2\% usa por $2 \mathrm{~h}$ a $3 \mathrm{~h}$ pela tarde, $18,4 \%$ faz uso por $1 \mathrm{~h}$ pela manhã e $36,9 \%$ utiliza por $2 \mathrm{~h}$ a $3 \mathrm{~h}$ à noite. Em relação à idade em que aprenderam a utilizar a internet, 57,4\% tinha a idade entre 6 e 10 anos, 24,4\% entre 11 e 13 anos, $11,3 \%$ tinha menos de 5 anos, $6 \%$ entre 13 e 15 anos, e $0,6 \%$ entre 16 e 18 anos.

Questionados a respeito de quem ensinou a utilizar a internet, 58,52\% afirmaram que aprenderam sozinhos, $17,99 \%$ com o pai, $13,63 \%$ com irmãos, $10,79 \%$ com a mãe, $10,79 \%$ com amigos, $8,14 \%$ fizeram curso.

A fim de avaliar como os adolescentes percebiam o uso de tal tecnologia de maneira prospectiva, perguntamos: "Tu acreditas que com o tempo as pessoas vão se comunicar mais por meio do computador do que pessoalmente?" A maioria dos sujeitos entrevistados $(80,7 \%)$ responderam afirmativamente.

A partir dos dados, podemos constatar que a maior parte dos adolescentes aprenderam a lidar com essa tecnologia já na primeira infância, antes dos 10 anos e de forma autodidata. Essa autonomia no aprendizado caracteriza uma geração de jovens precocemente familiarizados e, consequentemente, destemidos para dominar as novas tecnologias. Tal familiarização se deve à democratização do acesso favorecida por fatores de ordem sócio-econômica. Observa-se que nos últimos cinco anos (Ibope/ NetRatings, 2007) houve uma ampliação do acesso a internet de alta velocidade (banda larga), assim como subsídios governamentais que contribuíram para baratear essa tecnologia de maneira que se expandisse a todas as camadas da população. Neste processo, constata-se atualmente a paulatina diminuição de preços dos computadores e celulares, por exemplo. Nesse caso, fatores tais como o baixo preço, a desatualização rápida dos aparelhos e o fato de serem auto-explicativos e de fácil manejo, se por um lado, contribuem para que haja uma relativa descartabilidade de tais aparelhos, por outro, amplia as possibilidades de sua utilização.

\section{Vantagens e desvantagens}

Com a finalidade de investigar a opinião dos adolescentes quanto às vantagens da utilização da internet, solicitou-se que eles fizessem uma lista delas. Os resultados (descritivos) aparecem na Tabela 2 , (cabe ressaltar que as porcentagens somam mais de $100 \%$ porque cada adolescente listou mais de uma vantagem):

Tabela 2 - Vantagens do uso da internet

\begin{tabular}{ccc}
\hline $\begin{array}{c}\text { Vantagens do } \\
\text { uso da internet }\end{array}$ & Frequências & Porcentagens \\
\hline Rapidez & 377 & $70,99 \%$ \\
\hline Econômicas & 243 & $45,76 \%$ \\
Conhecer pessoas & 238 & $44,82 \%$ \\
\hline Aumentar o número de amigos & 206 & $38,79 \%$ \\
\hline Não precisa ver a pessoa & 110 & $20,71 \%$ \\
\hline Nenhuma & 35 & $6,59 \%$ \\
\hline
\end{tabular}

Fonte: Dados da pesquisa.

Assim como, em relação às desvantagens, eles listaram mais de um item conforme os resultados apresentados na Tabela 3:

Tabela 3 - Desvantagens do Uso da Internet

\begin{tabular}{ccc}
\hline $\begin{array}{c}\text { Desvantagens do uso da } \\
\text { imternet }\end{array}$ & Frequências & Porcentagens \\
\hline Pode viciar & 265 & $49,81 \%$ \\
\hline Pode ser perigoso & 256 & $48,12 \%$ \\
\hline Falta de contato com a pessoa & 229 & $43,04 \%$ \\
Limitações para se expressar & 204 & $38,34 \%$ \\
\hline Nenhuma & 56 & $10,52 \%$ \\
\hline
\end{tabular}

Fonte: Dados da pesquisa. 
Identifica-se, nos resultados anteriores, que a rapidez na comunicação é a vantagem mais apontada pelos jovens $(70,99 \%)$. 0 fato de ser uma tecnologia econômica e a possibilidade de conhecer pessoas e aumentar o número de amigos, são nomeados também com bastante incidência. Esses aspectos expressam algumas das características mais marcantes dos adolescentes chamados de "Geração Y", por causa da emergência que apresentam na busca de respostas, na praticidade que demandam daquilo que manejam em seu contexto e da utilização da internet como ferramenta importante na sua interação social. Nossos dados corroboram outros estudos realizados no contexto brasileiro (Almeida \& Eugênio, 2006; Nicolaci da Costa, 1998, 2002, 2006) que mostram a importância da internet no relacionamento social dos jovens, especialmente com seus iguais.

Entretanto apesar de os achados ilustrarem peculiaridades que abrangem uma geração de jovens, parece que as vantagens e as desvantagens são matizadas pelas características e circunstâncias de cada um. Isso se expressa no fato de que $20,71 \%$ dos adolescentes relatam como vantagem não precisar ver a pessoa com a qual estão se comunicando, entretanto 43,03\% apontaram como desvantagem a falta de contato com a pessoa no momento da comunicação, assim como que $38,34 \%$ disseram ser uma desvantagem a limitação para expressarem-se nesta interação virtual.

0 aspecto mais apontado pelos sujeitos entrevistados como sendo negativo na utilização de tal tecnologia, é a possibilidade de que seu uso vicie. Entretanto, se considerarmos o tempo de utilização declarado por eles, que não supera o número de $3 \mathrm{~h}$ diárias, parece que, efetivamente, os adolescentes desta amostra fazem um uso moderado da internet, talvez, em virtude da consciência que têm a respeito da possibilidade de viciar.

Essas nuances expressas nos dados nos fazem refletir que a comunicação virtual por si só não pode ser classificada como positiva ou negativa. Observa-se que o contexto social e familiar de cada jovem, assim como suas características pessoais, são variáveis importantes a serem consideradas na explicação de como cada jovem utiliza tal tecnologia.

\section{Frequência e locais de utilização}

Investigou-se a frequência e os locais onde os jovens fazem uso da internet. Por meio do Teste Mann-Whitney comparamos os resultados de meninos e meninas, conforme apresentamos na Tabela 4:
Tabela 4 - Frequência do uso da internet

\begin{tabular}{ccc}
\hline \multicolumn{3}{c}{ Frequência do uso internet (\%) } \\
\hline & Meninos & Meninas \\
\hline Com muita frequência & 54,1 & 53,1 \\
\hline Bastante & 33,5 & 27,4 \\
Moderadamente & 10,3 & 15,4 \\
\hline Pouco & 1,2 & 2,4 \\
\hline Muito pouco & 0,4 & 1,7 \\
\hline Total & 100 & 100 \\
\hline
\end{tabular}

Fonte: Dados da pesquisa.

Não foram verificadas diferenças significativas entre os sexos $(p=0,183)$. A grande maioria, mais de $80 \%$ dos meninos e das meninas, revelaram utilizar com bastante ou muita frequência a internet.

Quanto aos locais de uso, foram dadas as seguintes alternativas a fim de que eles avaliassem cada uma a partir de uma escala Likert de 5 pontos (desde nunca até sempre): em casa, na casa de amigos, na escola, na casa de outros familiares e em outros locais como lan-house, cafés, etc.

A maior parte dos jovens, responderam que sempre utilizam internet em casa, sendo 81,3\% dos adolescentes de escolas particulares, 70,4\% de escola federal e $67,1 \%$ de escolas estaduais. Já nas alternativas que compreendem locais fora de casa (casa de amigos, escola, lan-house, casa de outros familiares) as respostas variam de às vezes a nun$c a$, sendo que $34,7 \%$ dos adolescentes de escolas particulares disseram que às vezes utilizam a internet na casa de amigos, assim como $33,1 \%$ dos estudantes de escolas federais e $34,2 \%$ de escolas estaduais. Similarmente, $30,8 \%$ dos jovens de escolas federais responderam às vezes, em referência à utilização na casa de outros familiares, $25,3 \%$ dos de escolas estaduais marcaram nunca e $28,8 \%$ dos adolescentes de escolas particulares declararam raramente e às vezes.

A utilização na escola também apresentou essas características: 78,4\% dos adolescentes de escolas estaduais responderam nunca, 45,4\% dos de escola particular 40,0\%, dos jovens de escolas federais disseram que raramente acessam internet na escola. Por fim, a utilização em locais como lan-houses e cafés também mostrou ser uma rara opção para os adolescentes de escolas federais, $34,3 \%$, sendo que $37 \%$ e $43,3 \%$ dos entrevistados de escolas estaduais 
e particulares respectivamente, responderam nunca acessar a internet nestes locais.

Identifica-se a partir destas respostas que os adolescentes, predominantemente, utilizam a internet em suas casas, havendo menor frequência de acesso em outros locais. Estes resultados são interessantes, porque demonstram que a utilização da internet em casa é mais uma das atividades do cotidiano doméstico dos adolescentes. Nesse caso, assim como assistem à televisão, escutam música, os jovens atualmente passam de $2 \mathrm{~h}$ a $3 \mathrm{~h}$ do tempo em que estão em seus lares navegando no espaço virtual.

\section{As famílias e o uso da internet}

Quanto ao uso da internet e o contexto familiar dos jovens, foram investigadas as seguintes variáveis: local da casa onde está o computador, combinações existentes para a utilização da internet e a utilização que os progenitores fazem desta tecnologia.

Dentre os adolescentes investigados 52,85\% afirmaram não existir nenhum tipo de controle familiar na utilização que fazem da internet, sendo que $28,32 \%$ revelaram que os pais definem o horário de uso; $25,28 \%$ só permitem uso após as tarefas; 5,\% dizem que a família proíbe o computador no quarto; 2,09\% informaram que os pais usam filtros para bloquear sites, sendo que menos de $1 \%$ só permitem o uso acompanhado de algum responsável. A maioria dos jovens $(82,9 \%)$ informou que seus pais costumam utilizar a internet, sendo que 17,1\% não utiliza tal tecnologia.

Pode-se constatar que, segundo os adolescentes, existem dois grupos de famílias no que se refere ao controle do uso da internet: famílias que não fazem nenhum tipo de controle e famílias que utilizam uma diversidade de alternativas de controle. Dentre estas, as práticas variam desde métodos sofisticados como o uso de filtros, o que denota maior conhecimento por parte dos progenitores das ferramentas disponíveis para esse fim, até uma proibição total do uso desacompanhado. Associando-se aos resultados apresentados em relação aos locais de uso, em que identificamos que os jovens acessam a internet predominantemente em casa, fica evidente que há um grande número de famílias que não monitora seus filhos (as) adolescentes no espaço doméstico.

Esses resultados corroboram os achados da pesquisa realizada pela ONG SaferNet Brasil (2008), a qual identificou um índice ainda maior: $87 \%$ dos jovens e famílias pesquisadas também afirmaram não possuir nenhum tipo de controle quanto ao uso da internet pelos filhos adolescentes. Parece que, apesar de termos uma amostra de progenitores, predominantemente, com nível superior de escolaridade e que tem o hábito de utilizar a internet, há uma certa negligência em relação ao monitoramento de seus filhos na utilização que fazem dessa tecnologia.

\section{Compartilhar conhecimentos sobre a internet}

Perguntou-se aos adolescentes com quem compartilham as descobertas de coisas interessantes que podem ser feitas ou vistas na internet. As alternativas de respostas incluíam: o pai, a mãe, os irmãos, algum professor, amigos da mesma idade e amigos mais velhos, em uma escala likert de cinco pontos variando de nunca até sempre.

Um grande número de adolescentes, independente do sexo, conversa a respeito desses assuntos com os amigos da mesma idade $(45,5 \%$ das meninas disseram sempre e $37,6 \%$ dos meninos quase sempre). Assim como o fazem com amigos mais velhos, sendo $28,4 \%$ das meninas e $27,3 \%$ dos meninos que responderam quase sempre.

Em relação ao pai, 35,3\% das meninas disseram que nunca conversam com ele acerca de suas descobertas na internet e os $26,4 \%$ dos meninos disseram que às vezes o fazem. Em relação à mãe, 28,9\% dos meninos e $26 \%$ das meninas conversam às vezes. Os irmãos também são pouco procurados para conversar, $33,6 \%$ das meninas e $31,4 \%$ dos meninos nunca compartilham essas descobertas com os irmãos.

Por fim, os professores são os menos procurados pelos adolescentes para essas conversas, $66,9 \%$ dos meninos disseram que nunca o fazem assim como $65,4 \%$ das meninas.

Pode-se perceber, a partir destes resultados, que os adolescentes tendem a dividir suas descobertas em relação ao mundo virtual com seus iguais, dado que corrobora os achados de Casas, Figuer, Gonzáles e Malo (2007) que mostraram que os filhos não conversam com os pais sobre as novas tecnologias da informação, muitas vezes, porque acreditam que não compreendem este universo. Assim, utilizam a "ignorância" dos progenitores como justificativa para não compartilharem com eles o que fazem e descobrem na internet. 


\section{Considerações finais}

Esta pesquisa investigou o fenômeno da utilização da internet sob a perspectiva de adolescentes, geração a qual já nasceu convivendo com tal tecnologia. Nossos jovens caracterizam-se por terem sido precocemente expostos e estimulados ao aprendizado e uso dos equipamentos tecnológicos de informação e entretenimento.

A internet é um fato que já não podemos chamar de novo, entretanto a compreensão da sua forma de uso pelos adolescentes, principalmente, apresenta idiossincrasias quanto às reverberações desta prática dentro e fora do contexto familiar. Nossos achados permitiram um mapeamento, na perspectiva do próprio adolescente, de como se caracterizam algumas de suas práticas e a forma de lidar de suas famílias perante essa tecnologia. Identificamos que os adolescentes deste estudo iniciaram o uso da internet ainda na primeira infância, foram autodidatas, navegam no ciberespaço predominantemente em suas casas, embora, a maior parte das vezes, sozinhos e compartilham suas descobertas relacionadas ao mundo virtual com os amigos da mesma idade ou mais velhos que eles.

Tal perfil aponta algumas características familiares de como pais e mães têm lidado com as questões de monitoramento e autonomia com respeito a esta tecnologia. 0 mundo virtual parece ser algo do jovem, no qual os pais não participam ou participam pouco. Existe grande liberdade, desde o aprendizado de como utilizar, até mesmo a forma de como seguem utilizando no cotidiano doméstico. Diante disso, nos perguntamos: excesso de confiança na nova geração ou negligência quanto à exposição do filho(a) no mundo virtual?

A internet em si não pode ser considerada positiva ou negativa, assim como o uso que os jovens fazem desta tecnologia. Entretanto sabe-se que ela é uma janela para um mundo infinito de possibilidades, que podem ser enriquecedoras e favorecedoras de aprendizagem, mas também perigosas por causa da exposição do adolescente a uma série de ameaças virtuais que podem tornar-se reais. Talvez os pais ainda tenham pouco conhecimento a respeito dessa iminência, e, por isso, assumem uma atitude omissa e, por vezes, ingênua diante desse fenômeno.

A partir desse panorama, aponta-se a necessidade de uma maior participação e esclarecimento de pais, educadores e profissionais da saúde na relação dos jovens com essa tecnologia, visando a estimular o uso construtivo de tal ferramenta assim como fomentando a consciência das ameaças existentes no mau uso. 0 cuidado e a proteção dos filhos, nesse caso, ainda seguem sendo uma das tarefas mais antigas da família, mesmo perante às mais modernas tecnologias que o desenvolvimento das ciências possa oferecer à humanidade.

\section{Referências}

Almeida, M. I., \& Eugênio, F. (2006). 0 espaço real e o acúmulo que significa: Uma nova gramática para se pensar o uso jovem da internet no Brasil. In A. M. Nicolaci-da-Costa (Org.). Cabeças digitais: 0 cotidiano na era da informação (pp. 49-80). Rio de Janeiro: PUC-Rio.

Casas, F., Figuer, C., González, M., \& Malo. S. (2007). Los medios audiovisuales entre los progenitores y los hijos e hijas. Cultura y Educación, 19(3), 331-330.

Hollingshead, A. B. (1975). Four factor índex of social status. Departament of Sociology, Yale University. (Não publicado).

IBOPE/NetRatings. (2007). IBOPE/NetRatings divulga dados sobre o uso crescente da Internet. Recuperado em 17 out. 2007, em http://www.ibope.com.br/ calandraWeb/servlet/CalandraRedirect?temp $=6 \& \mathrm{p}$ roj=PortalIBOPE \&pub=T\&db=caldb\&comp=pesqui sa_leitura\&nivel=null \&docid=1946DA4AACE3A77B8 32572AB007278D0

IBOPE/NetRatings. (2008). Brasil superou o número de 40 milhões de pessoas com acesso à Internet. Recuperado em 25 out. 2008, em http://www.ibope. com.br/calandraWeb/servlet/CalandraRedirect? temp $=6 \&$ proj $=$ Porta

Malo, S. (2008). Cultures mediàtiques adolescents: Un estudi psicosocial centrat en el telèfon móbil. Girona. Tese de Doutorado, Institut de Recerca sobre Qualitat de Vida i Departament de Psicología, Universidade de Girona, Girona.

Music Television - MTV. (2005). Dossiê Universo Jovem 3. São Paulo: Brasil.

Nicolaci-da-Costa, A. M. (1998). Na malha da rede: Os impactos íntimos da internet. Rio de Janeiro: Campus. 
Nicolaci-da-Costa, A. M. (2002). Revoluções tecnológicas e transformações subjetivas. Psicologia: Teoria e Pesquisa, 18(2), 193-202.

Nicolaci-da-Costa, A. M. (2006). Cabeças digitais: 0 cotidiano na era da informação. Rio de Janeiro: PUC-Rio.

SaferNet Brasil. (2008). RELEASE: 87\% dos jovens afirmam que não possuem restrições para uso da Internet. Recuperado em 9 set. 2008, em http:// www.safernet.org.br/twiki/bin/view/SaferNet/ Noticia20081009202936
Tapscott, D. (1998). Growing up digital: The rise of the net generation. New York: McGraw-Hill.

Tudge, J. R. H., \& Frizzo, G. F. (2002). Classificação baseada em Hollingshead do nível sócio-econômico das famílias do estudo longitudinal de Porto Alegre: Da gestação à escola. Porto Alegre: Instituto de Psicologia, Universidade Federal do Rio Grande do Sul. (Não publicado).

Young, K. (1998). Caught in the net: How to recognize the signs of the internet addiction. New York: John Wiley \& Sons. 
